\title{
Evaluación de una intervención para la mejora de las competencias de regulación interpersonal del afecto y el bienestar laboral en una muestra de operadores penitenciarios del Uruguay
}

\section{Evaluation of a Training Program aiming to Improve Interpersonal Emotion Regulation Strategies and Occupational Wellbeing in a Uruguayan Prison}

\author{
David MARTÍNEZ-Í̃̃IGOa \\ Universidad Rey Juan Carlos, España \\ ORCID: http://orcid.org/0000-0002-2819-7883 \\ Antonio Crego \\ Universidad a Distancia de Madrid, España
}

${ }^{a}$ Correspondance author. e-mail: david.martinez@urcj.es

Para citar este artículo: Martínez-Iñigo, D., \& Crego, A. (2017). Evaluación de una intervención para la mejora de las competencias de regulación interpersonal del afecto y el bienestar laboral en una muestra de operadores penitenciarios del Uruguay. Universitas Psychologica, 16(3), 1-15. https://doi.org/10.11144/ Javeriana.upsy16-3.eimc

\section{RESUMEN}

Según estudios previos, el uso de estrategias controladas para la regulación interpersonal (ECRI) del afecto de otros orientadas a su mejora incrementa el bienestar de quien las emplea, mientras el uso de ECRI para empeorar el afecto lo deteriora. El consumo y recuperación de recursos de autoregulación de cada ECRI explican estos resultados. Mediante un diseño cuasiexperimental se evalúa la eficacia de la formación en ECRI para mejorar el bienestar en operadores de prisiones. Los resultados reflejan mayor bienestar y menor uso de ECRI de empeoramiento tras la formación $(\mathrm{N}=21)$, al comparar con el grupo control $(\mathrm{N}=18)$. No hubo diferencias para las ECRI de mejora. Los cambios en las ECRI de empeoramiento predicen negativamente cambios en el bienestar.

Palabras clave

calidad de vida; necesidades; atención integral; inclusión social; capacidad; discapacidad

\section{ABSTRACT}

Previous research show a positive relationship between using IARS to improve other people's affect and the level of wellbeing of the agent implementing them, whereas using IARS to worsen other's affect can worsen the agent's wellbeing. These results can be explained as the balance between the self-control resources drained and recovered by each IARS. We evaluate the efficacy of a training program to increase prison officers' wellbeing and focused on IARS. Results, based on a quasi-experimental design, revealed a higher level of wellbeing and a lower increased on worsening strategies among the training participants $(\mathrm{N}=21)$, compared with a control group $(\mathrm{N}=18)$. Results were not significant for improving strategies. Changes in the use of IARS negatively predicted changes in wellbeing.

Keywords 
Interpersonal emotion regulation; psychological wellbeing; selfregulation; conservation of resources; prison officers

\section{Antecedentes}

Trabajar con personas privadas de la libertad en instituciones penitenciarias implica el contacto frecuente con un grupo de población estigmatizado, en ocasiones en un ambiente con condiciones de higiene pobres, con niveles importantes de hacinamiento, en el que pueden producirse situaciones peligrosas, ligadas a la violencia verbal y física, y en el que tampoco es infrecuente la exposición a la muerte, relacionada algunas veces con conductas suicidas (Naciones Unidas, 2009). Junto a ello, el hecho de que una parte importante de la población reclusa padece trastornos mentales, enfermedades crónicas o problemas de adicción hace aún más compleja la labor de los operarios de prisiones (Baillargeon, Binswanger, Penn, Williams, \& Murray, 2009; Diamond, Wang, Holzer, Thomas, \& Anges, 2001; Lamb \& Weinberger, 1998).

Diferentes autores han definido este tipo de actividad laboral como "trabajo sucio" (Ashforth \& Kreiner, 1999; Douglas, 1996), y su realización supone un desafío para el cumplimiento de algunas de las funciones psicosociales de la propia actividad laboral, especialmente de aquellas que se relacionan con el bienestar y la salud general de los individuos. Por ejemplo, en este escenario, los trabajadores pueden experimentar dificultades para construir una identidad profesional positiva que mantenga su autoestima, incluso cuando su prestigio profesional pueda ser relativamente alto o la sociedad aplauda que "alguien", que no sea uno mismo, se ocupe de estas labores (Ashforth \& Kreiner, 1999).

De manera específica, en relación al trabajo en prisiones, se ha señalado que la exposición continuada a situaciones potencialmente estresantes podría asociarse a graves consecuencias para la salud de los trabajadores (Cheek \& Miller, 1983; Math, Murthy, Parthasarthy, Kumar, \& Madhusudhan, 2011). Entre ellas, estudios recientes como los de Kinman, Clements y Hart (2016a, 2016b),
Viotti (2016), Jin, Sun, Jiang, Wang y Wen (2016) o Bringas-Molleda, Fernández-Muñiz, Álvarez-Fresno, Martínez-Cordero y RodríguezDíaz (2015) han alertado sobre el elevado riesgo de sufrir síndrome de burnout y estrés laboral entre los operarios de prisiones, lo que supone una amenaza para su bienestar ocupacional. Incluso, algunas investigaciones han encontrado que hasta un tercio de estos trabajadores podría verse afectado por estas problemáticas (Kalra et al., 2016). Más aún, las consecuencias pueden afectar al trabajador más allá del ámbito estrictamente laboral, como lo muestran diversos estudios de problemas médicos y psicológicos, tales como trastornos psicosomáticos, problemas cardiovasculares, síntomas de depresión y ansiedad o un mayor riesgo de cometer intentos de suicidio o de autolesiones (Härenstam, Palm, \& Theorell, 1988; Harvey, 2014; Sui et al., 2014; Roberts, Jaremin, \& Lloyd, 2013; Stöver, 2017). Tales riesgos profesionales y los daños de naturaleza psicosocial asociados suponen una amenaza para la calidad de vida del operario; pero, además, conllevan un impacto psicosocial que se extiende más allá del propio trabajador, y que afecta sus relaciones interpersonales (por ejemplo, conflicto familiar y divorcio) y a la propia institución penitenciaria (como la disminución de la calidad de la atención que presta a la población reclusa) (Armstrong, AtkinPlunk, \& Wells, 2015; Lambert, Barton-Bellessa, \& Hogan, 2015; Lambert, Minor, Wells, \& Hogan, 2015).

\section{Trabajo sucio y regulación emocional}

Un elemento característico del trabajo sucio es la generación de emociones no deseadas, que pueden llegar a interferir con la ejecución del rol laboral (Ashforth \& Kreiner, 2002). Para evitar esta interferencia, las organizaciones desarrollan estrategias que permiten la regulación de esas emociones indeseadas sin alterar el status quo (Ashforth \& Humphrey, 1993). No obstante, algunas de estas estrategias pueden resultar nocivas para los individuos (Martínez-Íñigo \& Crego, 2010), ya que 
suponen una carga adicional de trabajo para el empleado. Por ejemplo, con frecuencia estas estrategias implican la supresión emocional, la reinterpretación o reencuadre de la situación y el ajuste de la experiencia y la expresión emocional a distintas normas. Los estudios realizados reflejan que todos estos procesos de regulación y control de las emociones tienen implicaciones sobre el bienestar laboral y la salud de los trabajadores (para una revisión, véase Hülsheger \& Schewe, 2011).

La mayor parte de las investigaciones previas se han centrado en los procesos de regulación de las emociones propias o regulación emocional intrapersonal. En los últimos años, sin embargo, un número creciente de estudios reflejan que los efectos de este tipo de regulación están condicionados por aspectos interpersonales (Martínez-Íñigo, Totterdell, Alcover, \& Holman, 2007) y que una parte importante de la regulación emocional tiene como objetivo el manejo adecuado de las emociones de otras personas, es decir, implica una regulación emocional interpersonal (Niven, Totterdell, \& Holman, 2009).

Avanzar en el conocimiento de la dimensión interpersonal supone uno de los retos más importantes en el estudio de la regulación emocional, dada la función social de las emociones (Little, Kluemper, Nelson, \& Gooty, 2012; Zaki \& Williams, 2013).

El presente estudio contribuye a esta ampliación del estudio de la regulación emocional en el trabajo, mediante un análisis del impacto que los cambios en las ECRI empleadas por los operadores de prisiones, derivados de la capacitación, tienen sobre su bienestar psicológico en el trabajo. A continuación, se presenta la evidencia previa en la que se basa la expectativa de que un cambio en la frecuencia de uso de las distintas ECRI derive en mejoras del bienestar psicológico.

\section{Regulación emocional interpersonal y salud laboral}

Una de las principales demandas del rol laboral de los operadores de prisiones tiene que ver con la supervisión y control del comportamiento de los reclusos. Debido a que las emociones ejercen un control importante sobre la conducta (Baumeister, Vohs, \& Tice, 2007), para realizar esta tarea los operadores pueden tratar de influir de forma consciente sobre las emociones de los reclusos, con la intención de promover la regulación de sus comportamientos en la dirección deseada. Por ejemplo, el operario puede recordarle a un interno las consecuencias negativas de una conducta para que el miedo inhiba la repetición de dicho comportamiento, o puede comentar alguna cualidad positiva del recluso para animarle a perseverar en una conducta deseada. Tal forma de regulación emocional se denomina regulación interpersonal del afecto controlada, y se define como el conjunto de acciones emitidas por un agente con la intención consciente de modificar la experiencia emocional de otro persona o diana (Niven, Totterdell, Stride, \& Holman, 2011).

Aunque el número de conductas a través de la cuales el agente puede intentar modificar los sentimientos de la diana es considerablemente amplio, estas pueden ser agrupadas en un conjunto reducido de categorías (Niven et al., 2009). Así, desde el punto de vista de sus efectos sobre el bienestar psicológico, se establecen dos grandes categorías. Por un lado, se encontrarían las estrategias controladas de regulación interpersonal (ECRI) que pretenden mejorar el afecto (MA) de la diana. Por otro, las ECRI orientadas al empeoramiento del afecto (EA) de la diana (Niven et al., 2011).

Los resultados de estudios previos reflejan que la implementación de estrategias de EA tienen un efecto negativo sobre el bienestar no solo de la diana, sino que también se observa ese mismo efecto en el agente (Martínez-Ínigo, Poerio, \& Totterdell, 2013; Niven, Holman, \& Totterdell, 2012; Niven, Totterdell, Holman, \& Headley, 2012). En un estudio pionero sobre el tema, Niven, Totterdell y Holman (2007) 
hallaron que el uso de estrategias de EA reducía el bienestar de los operadores de prisiones y dañaba la calidad de la relación con los reclusos. Resultados más recientes confirman que el uso de estrategias de EA ejerce una influencia negativa sobre el bienestar de los operarios de prisiones (Niven, Totterdell et al., 2012). Pero, aún más, un estudio reciente ha encontrado resultados similares cuando un individuo observa a otro emplear estrategias dirigidas al EA, sin ser él ni el agente ni la diana (Totterdell, Hershchovis, Niven, Reich, \& Stride, 2012).

En cuanto a los efectos del uso de estrategias de MA, los resultados sobre su relación con el bienestar no son tan consistentes. Algunos estudios reflejan que mejora el afecto del agente (Niven, Totterdell et al., 2012), mientras que otros no encuentran un efecto significativo sobre el bienestar laboral, medido como agotamiento emocional (Martínez-Íñigo, Mercado, \& Totterdell, 2015). En el caso de los operadores de prisiones, Niven, Totterdell et al. (2012) encontraron una relación positiva entre el uso de estrategias de MA y el bienestar psicológico de los operadores de prisiones. Es importante destacar que la evidencia empírica muestra la presencia de efectos derivados del uso de estrategias de MA por parte de los operadores de prisiones sobre la calidad de la relación con los internos, medida como el nivel de amistad y confianza percibida por los reclusos en la relación con el operador (Niven, Hollman et al., 2012).

Los resultados de los estudios que analizan las relaciones entre regulación emocional interpersonal y bienestar podrían explicarse atendiendo a un doble proceso, en el que intervienen las demandas de autorregulación implicadas en las ECRI y la recuperación de recursos activados o inhibidos por la implementación de estas estrategias.

Las ECRI se caracterizan como comportamientos orientados a la consecución de, al menos, un objetivo primario: el cambio del afecto de otra persona. En cuanto a comportamientos orientados a un objetivo, demandan un esfuerzo de autorregulación por parte del agente. Tales rasgos - la existencia de un objetivo primario y la implementación de comportamientos encaminados a su consecución - son definitorios del proceso de autorregulación (Vhos \& Baumeister, 2007). La puesta en marcha de una ECRI puede requerir el uso de estos recursos de autorregulación en diferentes momentos de su ejecución. Por ejemplo, el agente debe decidir qué estrategia pone en marcha, tiene que supervisar que la estrategia seleccionada es correctamente implementada o puede tener que decidir —en función de los resultados obtenidos- si debe cambiar de estrategia o de objetivo.

De acuerdo con el Modelo de la Fuerza de Autorregulación (Strength Model of Self-Control) (Baumeister et al., 2007), los recursos de los que dispone un individuo para regular su propio comportamiento son limitados, y cada acto de autorregulación reduce el nivel de recursos disponibles (Baumeister, Bratslavsky, Muraven, \& Tice, 1998). Por tanto, la ejecución de ECRI contribuye a la reducción de los recursos de autorregulación disponibles, lo que puede derivar en un estado de agotamiento o ego-depletion (Baumeister et al., 1998). De acuerdo con este modelo, las distintas ECRI se relacionarían negativamente con el bienestar, al incrementar los niveles de ego-depletion.

Sin embargo, estas predicciones no corresponden exactamente con los resultados encontrados hasta el momento, ya que la relación negativa con el bienestar tan solo ha sido confirmada en el caso de las estrategias EA, siendo esta nula o positiva en el caso de las estrategias de MA.

Para poder explicar los resultados obtenidos, es necesario incorporar los procesos de recuperación de recursos. Los estudios sobre autorregulación ponen de manifiesto que dichos recursos pueden ser restablecidos por medio del descanso, la relajación o la ingesta de glucosa (Gailliot et al., 2007; Tyler \& Burns, 2008). Así, la explicación a las divergencias en los efectos que las diferentes ECRI tienen sobre el bienestar se explicarán a partir de los efectos diferenciales que las estrategias de EA y de MA tienen sobre alguno de los mecanismos de recuperación de recursos de autorregulación. En concreto, sobre los procesos de recuperación 
asociados a la retroalimentación procedente de la diana y que recibe el agente. De acuerdo con el Modelo de Conservación de Recursos, el establecimiento de relaciones positivas con otras personas permite recuperar recursos y reducir la tensión (Hobfoll, 1989). En este sentido, el establecimiento de este tipo de relaciones reduce los efectos negativos de la regulación emocional sobre el bienestar del trabajador (Brotheridge \& Lee, 2002; Martínez-Íñigo et al., 2007). En particular, Martínez-Íñigo et al. (2007) han encontrado que la retroalimentación aportada por las dianas - pacientes en un contexto sanitario, en este estudio- media la relación entre las estrategias de regulación emocional y el agotamiento emocional.

Aplicando este esquema a las ECRI, parece razonable que las probabilidades de obtener retroalimentación favorable por parte de la diana, tras una estrategia de EA, sean bajas. Por el contrario, las probabilidades de obtener retroalimentación favorable serán mayores al implementar una estrategia de MA. Por tanto, las estrategias de EA no permitirían la recuperación de recursos derivados de relaciones positivas, mientras que las estrategias de MA contribuirían a la recuperación por medio de la retroalimentación favorable de la diana.

La ausencia, en el caso del EA, o la presencia, en el caso de la MA, de este mecanismo de recuperación de los recursos empleados en la autorregulación explicaría por qué, a pesar de que todas las ECRI conllevan un consumo de recursos, unas - las EA - se relacionan negativamente con el bienestar y otras - las MA - lo hacen de forma positiva o nula.

Estudios experimentales recientes en el campo de la regulación emocional intra e interpersonal demuestran que el equilibrio entre el consumo y la recuperación de recursos forma parte de la explicación del impacto de la regulación emocional sobre el bienestar psicológico (Grandey, Chi, \& Diamond, 2013; Martínez-Íñigo et al., 2015).

| Universitas Psychologica | V. i6 | No.3 | 2017 |

\section{Mejora de las competencias de regulación emocional y bienestar laboral}

A pesar de que un número creciente de estudios apoyan la relevancia del uso de ECRI para el bienestar de los empleados, no existe, hasta dónde los autores conocen, ninguna evidencia empírica sobre la posibilidad de modificar dicho uso mediante programas de formación estandarizados ni sobre el impacto que se deriva de estos programas para el bienestar laboral de los participantes.

De acuerdo con el modelo teórico descrito y los resultados previos, este estudio pretende contribuir a determinar si los cambios en la frecuencia de uso de las distintas ECRI, derivados de la implementación de un programa orientado a mejorar las competencias de regulación emocional interpersonales, se relaciona de forma significativa con cambios en los niveles de bienestar laboral de los participantes en la formación. Para ello, se utilizó un diseño cuasiexperimental, en el que se incluía un grupo experimental o de formación y un grupo control. Los resultados del estudio son relevantes tanto desde el punto de vista teórico como empírico y práctico. Por un lado, desde una perspectiva conceptual, contribuyen a la caracterización de las ECRI como habilidades susceptibles de entrenamiento. Por otro lado, suponen una ampliación de la evidencia empírica sobre la relación entre las ECRI y el bienestar psicológico de forma práctica y ofreciendo una nueva vía para la mejora del bienestar laboral en aquellas ocupaciones dónde la interacción con otras personas constituye una pieza clave. En el caso de ocupaciones donde es común que exista cierta tensión y conflicto en la relación - como es el caso de los operadores de prisiones, que son responsables de la supervisión de la vida cotidiana de personas privadas de la libertad-, el desarrollo de herramientas para la mejora de su bienestar resulta especialmente relevante. Más aún si esto pasa por el desarrollo de estrategias (ECRI de mejora del afecto) que los estudios previos en prisiones demuestran que tienen un impacto positivo sobre ambas partes en la relación —operador e interno—, así como sobre 
la calidad de su relación (Niven, Hollman et al., 2012).

El estudio plantea las siguientes hipótesis:

H1: El nivel de bienestar psicológico tras la formación será significativamente mayor en el grupo de formación comparado con el grupo de control.

H2: El nivel de uso de estrategias de MA tras la formación será significativamente mayor en el grupo de formación comparado con el grupo de control.

H3: El nivel de uso de estrategias de EA tras la formación será significativamente menor en el grupo de formación comparado con el grupo de control.

H4a: Para el conjunto de la muestra, los incrementos en las estrategias de MA predecirán positivamente los cambios en la salud general.

H4b: Para el conjunto de la muestra, los incrementos en las estrategias de EA predecirán negativamente los cambios en la salud general.

\section{Método}

\section{Participantes}

Un total de 39 operadores de prisiones uruguayos participaron en el estudio, con una media de edad de 31.4 años $(\mathrm{Dt}=9.5)$. El estudio se realizó en dos centros penitenciarios para reclusos varones y en uno destinado al internamiento de mujeres, en Uruguay. El $61.5 \%$ de los participantes eran hombres. En cuanto al nivel de estudios de los participantes el $7.7 \%$ tenía estudios primarios, el $28.2 \%$ había concluido estudios secundarios básicos, el $48.7 \%$ alcanzó el bachillerato y el 10.3 \% había iniciado estudios universitarios. La antigüedad media en el puesto fue de 14.85 (Dt= 9.01).

\section{Procedimiento y diseño}

La evaluación de la eficacia del programa de formación se llevó a cabo mediante un diseño cuasiexperimental, en el que los participantes se distribuyeron en un grupo experimental, que recibía el programa de formación, y un grupo control, al que no se administró ninguna intervención. Un total de 21 operadores, seleccionados por los directores de los centros, pasaron por el programa de formación. Para recoger la información del grupo de control, se pidió a los participantes que uno de los operadores con los que trabajaban habitualmente completara el cuestionario, procedimiento que favorece la similitud entre los integrantes de los grupos experimental y control. En tres de los casos no fue posible obtener esta información, por lo que el grupo control quedó compuesto por 18 operadores. Tanto en el grupo de formación como en el de control la participación en el estudio era voluntaria y sin contraprestación alguna.

Se tomaron medidas, tanto en el grupo experimental como en el de control, en los momentos anterior (T1) y posterior (T2) a la realización del programa de formación. Las variables de control fueron medidas en el pretest (T1), mientras que las variables referentes al uso de estrategias de regulación emocional y al bienestar psicológico se midieron en T1 y T2.

El programa de formación constaba de 10 sesiones de aproximadamente una hora y media de duración cada una, que eran impartidas por dos formadores en cada uno de los centros participantes. Estos formadores habían sido entrenados con anterioridad para llevar a cabo sus funciones, mediante una preparación especializada de 180 horas de duración impartida por el equipo de investigación en la Facultad de Psicología de la Universidad de la República de Uruguay. Además, se les proporcionaron materiales estandarizados y diseñados específicamente para el programa de formación de operarios, como fichas para cada sesión, pautas para realizar ejercicios prácticos y presentaciones de diapositivas por ordenador.

El programa de formación que se administró a los operarios de prisiones tenía un carácter fundamentalmente práctico, articulándose a través de una serie de ejercicios basados en técnicas y dinámicas de grupo que provienen del enfoque cognitivo-conductual, cuya eficacia se había contrastado previamente en otros contextos (Martínez-Íñigo, Totterdell, Alcover, 
\& Holman, 2009). Estas técnicas fueron adaptadas por el equipo investigador para su uso en el contexto penitenciario. El programa de formación presentaba, además, un marcado carácter psicoeducativo, tratando de hacer accesibles a los participantes los fundamentos de cada uno de los ejercicios, así como proporcionar información precisa y adecuada sobre los riesgos psicosociales a los que se ven expuestos los operarios de prisiones y la forma más saludable de afrontarlos (véase Tabla 1).

\section{TABLA 1}

Contenidos y objetivos del programa de entrenamiento desarrollado en prisiones

\begin{tabular}{ll}
\hline Sesión & Contenidos \\
\hline 1 & Preparación, motivación e iniciación del entrenamiento \\
\hline 2 & $\begin{array}{l}\text { Identificación de riesgos psicosociales en el entorno de prisiones y } \\
\text { motivación hacia el autocuidado }\end{array}$ \\
\hline 3 & $\begin{array}{l}\text { Comprensión y análisis de contextos potencialmente estresantes. El } \\
\text { papel de la empatia en el afrontamiento de situaciones }\end{array}$ \\
\hline 5 & $\begin{array}{l}\text { Identificación de emociones. Manejo de la ansiedad } \\
\text { manejo de pensamientos negativos }\end{array}$ \\
\hline 6 & $\begin{array}{l}\text { Habilidades interpersonales de regulación emocional y } \\
\text { comunicación. Estilos comunicacionales }\end{array}$ \\
\hline 7 & $\begin{array}{l}\text { Comprensión y manejo de los mecanismos implicados en la } \\
\text { agresividad }\end{array}$ \\
\hline 9 & Prácticas de manejo de la agresividad (I) \\
\hline 10 & $\begin{array}{l}\text { Autocuidados, recuperación emocional de manejo de la agresividad (II) } \\
\text { contexto penitenciario }\end{array}$ \\
\hline
\end{tabular}

Fuente: elaboración propia.

\section{Instrumentos}

Variables de control. En los distintos análisis realizados, se controlaron los efectos del género y de la afectividad positiva y negativa. Para evaluar la afectividad positiva, se aplicó la versión en español (Sandín, et al. 1999) del Positive and Negative Affect Schedule (PANAS), compuesta por 10 ítems relativos a estados afectivos negativos y 10 ítems que recogen estados afectivos positivos. Se pidió a los participantes que indicaran en una escala de 5 puntos (1= Nunca; 5 = Muy frecuentemente) con qué frecuencia experimentaban cada una de las emociones negativas (p. ej., "culpa" o "temor") y positivas (p. ej., "orgullo", "entusiasmo") que componían los ítems. Los valores del índice de consistencia interna fueron satisfactorios para la afectividad positiva y negativa (alpha $=0.86 \mathrm{y}$ alpha $=0.8$, respectivamente).

Estrategias Interpersonales de Regulación Emocional. Las estrategias de MA y de EA se midieron con la Escala de Regulación de las Emociones de Otros (Niven, et al., 2011). Se pidió a los participantes que indicaran en qué medida habían utilizado cada una de las seis estrategias de mejora del afecto de otros (ítem de muestra: "Escuché los problemas de un recluso para que se sintiera mejor") y cada una de las seis estrategias de EA (ítem de muestra: "Me hice el enfadado con un recluso para que se sintiera peor") durante las últimas dos semanas. La escala tiene un formato de respuesta tipo Likert con 5 puntos ( $1=$ nunca; $5=$ muy frecuentemente). Los índices de consistencia interna para las estrategias de mejora en T1 y T2 (alpha= 0.8 y alpha $=0.95$, respectivamente) y para las de empeoramiento fueron satisfactorios (alpha $=$ 0.75 y alpha $=0.93$, respectivamente) .

Bienestar psicológico. Se evaluó mediante la versión en castellano (Cifre \& Salanova, 2000) del Cuestionario de Salud General (GHQ; Goldberg, 1992). La escala está compuesta por 12 ítems (p. ej., "Sus preocupaciones le han hecho perder mucho el sueño"). Cada ítem presenta un formato de respuesta tipo Likert con cuatro opciones de respuesta. Los índices de consistencia internar fueron aceptables en T1 y T2 (alpha $=0.86$ y alpha $=0.91$, respectivamente)

\section{Resultados}

En la Tabla 2 se recogen los estadísticos descriptivos para las variables de estudio en cada uno de los momentos en que fueron evaluadas.

Para analizar si se observaban cambios significativos tras la intervención en las variables evaluadas, se realizaron tres análisis de covarianza (ANCOVA) mixtos de dos factores. 
En cada uno de ellos se tomaron como variables dependientes, respectivamente, las estrategias interpersonales orientadas a la mejora del afecto, las estrategias interpersonales orientadas a empeorar el afecto y el nivel de bienestar psicológico. En todos ellos, se controlaron los efectos de la afectividad positiva y negativa y el género.

\section{TABLA 2}

Estadísticos descriptivos (medias y desviaciones típicas) en T1 y T2 para los grupos experimental y control

\begin{tabular}{|c|c|c|c|c|}
\hline \multirow[b]{2}{*}{ Variable } & \multicolumn{2}{|c|}{ Grupo experimental } & \multicolumn{2}{|c|}{ Grupo control } \\
\hline & Media & $D t$ & Media & $D t$ \\
\hline \multicolumn{5}{|l|}{ Tiempo I } \\
\hline 1. Afectividad positiva & 3.91 & 0.5 & 3.92 & 0.63 \\
\hline 2. Afectividad negativa & 2.26 & 0.58 & 2.14 & 0.64 \\
\hline 3. Mejora del afecto & 3.74 & 0.8 & 3.03 & 1.1 \\
\hline 4. Empeoramiento afecto & 1.32 & 0.52 & 1.44 & 0.52 \\
\hline 5. Salud general & 3.15 & 0.51 & 3.27 & 0.47 \\
\hline \multicolumn{5}{|l|}{ Tiempo 2} \\
\hline 6. Mejora del afecto & 3.8 & 0.85 & 2.71 & 1.10 \\
\hline $\begin{array}{l}\text { 7. Empeoramiento del } \\
\text { afecto }\end{array}$ & 1.24 & 0.32 & 1.84 & 1.13 \\
\hline 8. Salud general & 3.49 & 0.41 & 3.05 & 0.38 \\
\hline
\end{tabular}

Fuente: elaboración propia.

Los análisis reflejan que no existían diferencias significativas entre los grupos control y de formación para la afectividad positiva $\left(F_{1.31}=\right.$ $0.444 ; p=0.51)$ ni para la afectividad negativa $(F$ $1.31=0.367 ; p=0.548)$. En cuanto a las variables sociodemográficas, no se apreciaron diferencias significativas en la edad $\left(F_{1.38}=0.28 ; p=0.64\right)$ entre el grupo control (Media $=32.2 ; D t=10.2)$ y el de formación (Media $=30.8 ; D t=9.2)$.

La primera de las hipótesis enunciadas (H1) planteaba que, para poder afirmar la eficacia de la intervención, el incremento en los niveles de bienestar desde T1 a T2 debería ser mayor en el grupo experimental, en comparación con el grupo control. En el caso de los niveles de bienestar psicológico, los efectos de interacción entre la condición y el tiempo fueron significativos $\left(F_{1.31}=15.791 ; p<0.001 ; \eta^{2}=\right.$ 0.34) (Figura 1). Las comparaciones post-hoc no reflejan diferencias significativas en los niveles de bienestar psicológico entre ambos grupos en el T1 ( $\left.F_{1.31}=0.265 ; \mathrm{p}=0.610 ; \mathrm{d}=-0.24\right)$; mientras que el nivel de salud general del grupo de formación es significativamente mayor cuando se compara con el grupo control en T2 $\left(F_{1.31}=\right.$ 13.938; $p=0.001, d=3.49)$. En cuanto a la comparación, dentro de cada condición, de los niveles de bienestar en T1 y T2, se apreció un incremento estadísticamente significativo en la salud general de los participantes en la formación $\left(F_{1.31}=12.673 ; p=0.001 ; d=0.74\right)$, mientras que en el grupo control $\left(F_{1.31}=4.889 ; p=0.035\right.$; $d=-0.51$ ) se observaba un deterioro, también estadísticamente significativo, de los niveles de salud general. Los resultados confirman la eficacia del programa para la mejora del bienestar de los participantes (H1).

Figura 1.

Efectos de interacción entre tiempo y condición para la variable bienestar psicológico (GHQ)

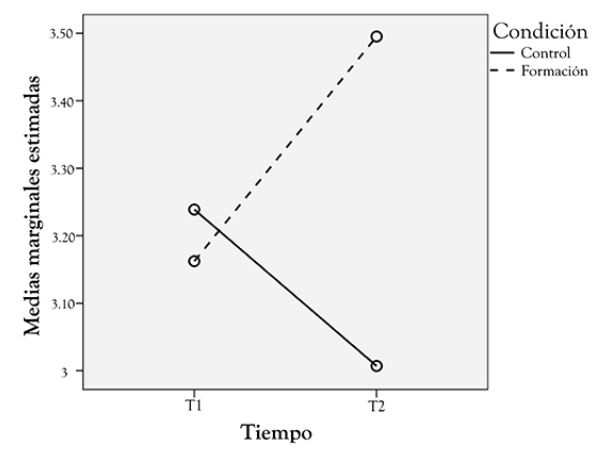

Fuente: elaboración propia.

En cuanto a la variación en el uso de estrategias de regulación interpersonal para mejorar el afecto del recluso, el término de interacción tiempo vs condición no fue significativo $\left(F_{132}=1.751, p=0.19\right)$ por lo que la $\mathrm{H} 2$, que predecía un patrón de cambio diferente en los grupos experimental y control en lo referente al uso de estas estrategias, no fue confirmada.

Los efectos de interacción entre el tiempo y la condición sí fueron, en cambio, significativos para las estrategias interpersonales orientadas a empeorar el afecto del recluso $\left(F_{1.31}=4.713\right.$; $\left.p=0.038 ; \eta^{2}=0.13\right)$, confirmando la $\mathrm{H} 3$, en la que se predecía un patrón de cambio diferente en el uso de estas estrategias en las condiciones experimental y control (Figura 2). 


\section{Figura 2.}

Efectos de interacción entre tiempo y condición para la variable uso de estrategias interpersonales de empeoramiento afecto

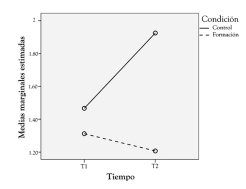

Fuente: elaboración propia.

Las comparaciones múltiples post-hoc reflejan que ambos grupos, control y formación, no mostraban diferencias significativas en el uso de este tipo de estrategias al inicio de la formación (T1) $\left(F_{1.31}=0.854 ; p=0.36 ; d=0.74 ;\right)$. Sin embargo, los niveles en esta estrategia en el T2 fueron significativamente menores en el grupo de formación que en el grupo control $(F$ $1.31=8.24 ; p=0.007 ; d=-0.72$ ) tal y como se esperaba. La comparación intragrupos reflejó que en el grupo de formación los niveles de uso de estrategias orientadas a empeorar el afecto de los reclusos disminuyeron de T1 a T2, si bien esta diferencia no fue estadísticamente significativa $\left(F_{1.31}=0.378 ; p=0.54 ; d=-0.18\right)$. No obstante, hay que remarcar que, en el mismo intervalo (T1 a T2), el grupo de control había incrementado significativamente la frecuencia de uso de estrategias orientadas a empeorar el afecto de los reclusos $\left(F_{1.31}=5.736 ; p=0.023 ; d=\right.$ 0.45).

Para confirmar si los cambios significativos en las estrategias interpersonales orientadas al empeoramiento del afecto del recluso se relacionan con las variaciones en los niveles de salud general, se realizaron análisis de regresión jerárquica. Al igual que en los análisis precedentes, se controlaron los efectos de la afectividad positiva y negativa y el género. Los resultados reflejan que la utilización de estrategias de empeoramiento del afecto del recluso en $\mathrm{T} 2$ predicen significativamente el nivel de bienestar psicológico en T2 $(\beta=-0.326$; $p=0.001)$ una vez controlados los efectos del nivel de bienestar psicológico inicial y la utilización de estas estrategias en T1 (Tabla 3). Los resultados confirman que los incrementos en el uso de estrategias EA del recluso predicen decrementos en la salud general del operador (H4b). Al no confirmarse la hipótesis sobre el incremento del uso de estrategias de mejora del afecto (H2), no se realizaron los análisis de regresión para este tipo de estrategia $(\mathrm{H} 4 \mathrm{a})$.

\section{TABLA 3}

Uso de estrategias interpersonales de empeoramiento del afecto de otros como predictor del bienestar psicológico $(n=35)$

\begin{tabular}{lll}
\hline Predictores & Paso 1 & Paso 2 \\
\hline Afectividad positiva & -0.24 & -0.03 \\
Afectividad negativa & -0.13 & 0 \\
Género & 0.21 & 0.19 \\
GHQ en T1 & 0.39 & $0.36^{*}$ \\
Empeoramiento afecto en T1 & 0.04 & 0.24 \\
Empeoramiento afecto en T2 & & $-0.62^{* *}$ \\
$\Delta R^{2}$ en cada paso & 0.08 & 0.38 \\
\hline
\end{tabular}

Fuente: elaboración propia.

\section{Conclusiones}

Los resultados del estudio apoyan la conveniencia de implementar estrategias formativas para mejorar la salud ocupacional de los operarios de prisiones, a través de la mejora de sus competencias emocionales. El bienestar psicológico de los participantes, tras la formación, fue significativamente mejor que el de los integrantes del grupo control. Además, el bienestar psicológico de aquellos que pasaron por el entrenamiento se incrementó significativamente, mientras que, en el mismo intervalo de tiempo, el bienestar en el grupo control se deterioró significativamente. Se puede afirmar, por tanto, que la formación tuvo al menos un efecto potenciador del bienestar de los participantes. Coherentemente con las conclusiones de Steiner y Wooldredge (2015), los resultados de la intervención servirían para ejemplificar que se puede intentar reducir el estrés de los operarios de prisiones aumentando su sensación de control sobre el trabajo, en 
este caso a través de un programa de formación centrado en los aspectos emocionales implícitos en él.

Respecto a las ECRI, la frecuencia de uso de estrategias de empeoramiento del afecto tras el entrenamiento fue menor en el caso del grupo de formación, con respecto al grupo de control. Se puede afirmar, por tanto, que la formación fue efectiva en la contención de los niveles de estrategias de empeoramiento del afecto negativas para el bienestar psicológico- en un contexto en el que dichos niveles estaban aumentando, como se aprecia en la evolución del grupo control.

Desde el punto de vista de la caracterización de las estrategias de regulación, pueden extraerse algunas consideraciones de carácter teórico sobre el fenómeno que pueden servir de objetivo para futuros estudios. La primera y más obvia, aunque hasta el momento no confirmada empíricamente, es que las estrategias de regulación emocional, al menos en algunas de sus dimensiones, son susceptibles de aprendizaje y, por tanto, de modificación. Las limitaciones de la muestra en el estudio no permiten analizarlo, pero futuros trabajos deberán tomar en consideración qué factores se relacionan con dichos cambios. Algunos podrían tener su origen en las características del propio individuo, otros relacionarse con las características del equipo, tal y como reflejan investigaciones recientes sobre la regulación emocional intrapersonal (Diefendorff, Erickson, Grandey, \& Dahling, 2011), y, otros pueden derivar de la propia organización y su cultura (Ashforth \& Kreiner, 2002). El desarrollo de modelos sobre ECRI que incluyan sus antecedentes supondría una contribución significativa y fructífera desde el punto de vista de la investigación.

Otro elemento interesante para el análisis de las ECRI tiene que ver con el patrón de cambios experimentados tras la formación. En este sentido, parece que la reducción en la frecuencia de uso de estrategias de empeoramiento fue más exitosa que la potenciación de las estrategias de mejora. Este resultado, si bien podría ser incidental y deberá ser replicado por futuros estudios, podría estar reflejando la naturaleza intrínsecamente interpersonal del fenómeno. Esto es, la estrategia seleccionada por el agente dependerá, en parte, de la respuesta que se espera emita la diana. En un contexto de trabajo donde existe una tensión entre ambas partes, como es el caso de la relación de custodia entre el operador penitenciario y el interno, es posible que resulte más fácil inhibir estrategias negativas que implementar estrategias positivas sobre las que no se tiene certeza de reciprocidad. En un estudio reciente, en el campo de la regulación de emociones intrapersonales se demuestra cómo este tipo de estimaciones sobre la reciprocidad en los resultados de una estrategia son relevantes en lo relativo a las consecuencias para el bienestar y, potencialmente, para la selección de estrategias de regulación en futuros encuentros (MartínezÍñigo \& Totterdell 2016). En este sentido, sería conveniente incluir esta dimensión en los modelos sobre ECRI y relaciones interpersonales.

Desde el punto de vista práctico, este patrón de cambios sugeriría la necesidad de intervenciones con ambas partes en la interacción, incluyendo el desarrollo de expectativas positivas sobre el feedback que el agente recibirá de la diana en caso de emplear estrategias de mejora.

Otra de las contribuciones del estudio deriva de los resultados que conectan empíricamente las variaciones en las ECRI con el bienestar psicológico, aportando información sobre los motivos por los que se observan mejoras. En este sentido, el estudio muestra que los incrementos en el uso de ECRI dirigidas a empeorar el afecto predicen decrementos en el bienestar psicológico. Recientemente se ha puesto de manifiesto la conveniencia de desarrollar estrategias de intervención centradas en la recuperación de los recursos empleados en la atención de las demandas propias del rol laboral. Este estudio, en línea con otros precedentes (Martínez-Íñigo et al., 2015; Muraven \& Baumeister, 2000), identifica los procesos de regulación emocional como procesos de autorregulación que consumen recursos limitados, cuyo agotamiento deriva en un deterioro del bienestar psicológico. Este tipo de procesos resulta de especial relevancia en aquellas ocupaciones donde las demandas 
emocionales son elevadas, como es el caso de los operadores de prisiones. Futuros estudios deberán profundizar en la evaluación de la eficacia de las intervenciones en la reducción del consumo o en la recuperación de los recursos de autorregulación, para así avanzar un paso más en la compresión de los mecanismos que explican la mejora del bienestar psicológico. Toda esta información, además de teóricamente relevante, es crucial para progresar en el desarrollo de estrategias cada vez más potentes y capaces de incrementar el tamaño de los efectos sobre el bienestar.

En relación con lo anterior, es necesario también tomar en consideración que, en determinados contextos - como por ejemplo la relación profesional de la salud-paciente, fuerza de seguridad-delincuentes u operarios de prisióninternos - la posibilidad de recibir una respuesta positiva tras una ECRI de mejora del ánimo es baja y, por tanto, existe un riesgo para el bienestar del profesional. De acuerdo con el modelo de intercambio social dual (Schaufeli, van Dierendonck, \& van Grop, 1996), la presencia de otras fuentes de reconocimiento, como por ejemplo los superiores inmediatos o las políticas de gestión de recursos humanos, pueden contribuir a compensar la falta de reciprocidad de la diana, reduciendo el riesgo. Por tanto, los futuros modelos sobre la utilización de las ECRI deberán incorporar este tipo de variables más allá del nivel puramente interpersonal, tal y como han hecho estudios recientes en el campo de la regulación emocional intrapersonal (Bechtoldt, Welk, Zapf, \& Hartig, 2013).

Es importante destacar también que el enfoque adoptado permite integrar dos vías de intervención, aquella que toma como referente al operario y la que adopta como objetivo al recluso. En relación con la primera, los resultados muestran una mejora en el bienestar psicológico del operario. Respecto a la segunda, dicha mejora procede de una reducción de conductas orientadas a empeorar el afecto del interno. Esta reducción sin duda tendrá un efecto positivo sobre la relación entre el operario y el recluso, tal y como reflejan estudios previos (Niven et al.,
2007), y cabe esperar también sobre el bienestar del interno.

En cuanto a las limitaciones del estudio, es importante destacar que la muestra empleada es pequeña, lo que podría explicar que algunos de los cambios encontrados en las ECRI y en el bienestar psicológico, aun siendo en la dirección esperada, no fueran significativos. La réplica del estudio con muestras mayores será necesaria para comprobar este aspecto.

Otra limitación importante tiene que ver con que la asignación de los participantes a las condiciones no fue aleatoria, por lo que podrían existir terceras variables que explicaran los resultados. No obstante, los análisis no reflejan diferencias significativas entre los grupos de formación y control en T1. La utilización de diseños experimentales en futuros estudios permitirá determinar las limitaciones derivadas del diseño aplicado en este estudio. En relación con el diseño, también sería recomendable que estudios posteriores incluyeran medidas de seguimiento para determinar la persistencia de los efectos encontrados.

A pesar de las limitaciones encontradas, el presente estudio supone una contribución significativa, especialmente por avanzar en la vinculación entre algunos de los desarrollos más recientes en el ámbito de la salud ocupacional y el abordaje de problemas organizacionales relevantes, como los que se manifiestan en los contextos penitenciarios uruguayos y de otros tantos países. Se suma así a un creciente número de estudios que, de manera notable y especialmente en el contexto de América Latina, han analizado la presencia de riesgos laborales de carácter psicosocial entre los funcionarios de prisiones (Clemente, Reig-Botella, \& Coloma, 2015; Fuertes, Moreno, \& Urquiola, 2017; Rodríguez Amaya, 2014). Pero, además, la intervención realizada para prevenir algunos de esos riesgos viene a dar respuesta a la sugerencia de autores como Stöver (2017) o Clemente et al. (2015), que recientemente han planteado la necesidad de implantar programas de reducción del estrés y promoción de la salud entre los trabajadores de prisiones. 


\section{Agradecimientos}

Los autores agradecen apoyo de la Agencia Española de Cooperación Internacional para el Desarrollo, Ministerio de Asuntos Exteriores y Cooperación, Gobierno de España, a través de la financiación del Proyecto de Cooperación Interuniversitaria A1/035232/11.

Igualmente agradecen la colaboración de la Facultad de Psicología de la Universidad de la República de Uruguay en la implementación del estudio a través del Centro de Investigación en Psicología de la Salud Ocupacional, Innovación y Cambio Organizacional (CIPSOICO), y al Ministerio de Interior de la República de Uruguay por facilitar el acceso a los participantes en el estudio

\section{Referencias}

Armstrong, G. S., Atkin-Plunk, C. A., \& Wells, J. (2015). The relationship between workfamily conflict, correctional officer job stress, and job satisfaction. Criminal Justice and Behavior, 42(10), 1066-1082.

Ashforth, B. E., \& Humphrey, R. H. (1993). Emotional labor in service roles: The influence of identity. Academy of Management Review, 18, 88-115.

Ashforth, B. E., \& Kreiner, G. E. (1999). "How can you do it?": Dirty work and the challenge of constructing a positive identity. Academy of Management Review, 24, 413-434.

Ashforth, B. E., \& Kreiner, G. E. (2002). Normalizing emotion in organizations: Making the extraordinary appear ordinary. Human Resource Management Review, 12, 215-235.

Baillargeon, J., Binswanger, I. A., Penn, J. V., Williams, B. A., \& Murray, O. J. (2009). Psychiatric disorders and repeat incarcerations: The revolving prison door. American Journal of Psychiatry, 166, 103-109.

Baumeister, R. F., Bratslavsky, E., Muraven, M., \& Tice, D. M. (1998). Ego depletion: Is the active self a limited resource? Journal of Personality and Social Psychology, 74(5), 1252-1265.

Baumeister, R. F., Vohs, K. D., \& Tice, D. M. (2007). The strength model of self-control. Current Directions in Psychological Science, 16, 351-355.

Bechtoldt, M. N., Welk, C., Zapf, D., \& Hartig, J. (2013). Main and moderating effects of self-control, organizational justice, and emotional labour on counterproductive behaviour at work. European Journal of Work and Organizational Psychology, 16, 479-500.

Bringas-Molleda, C., Fernández-Muñiz, A., Álvarez-Fresno, E., Martínez-Cordero, A., \& Rodríguez-Díaz, F. J. (2015). Influencia del síndrome de Burnout en la salud de los trabajadores penitenciarios. Revista Española de Sanidad Penitenciaria, 17(3), 67-73.

Brotheridge, C. M., \& Lee, R. T. (2002). Testing a conservation of resources model of the dynamics of emotional labor. Journal of Occupational Health Psychology, 7, 57-67.

Cheek, F., \& Miller, M. (1983). The experience of stress for correction officers: A doublebind theory of correctional stress. Journal of Criminal Justice, 11, 105-120.

Cifre, E., \& Salanova, M. (2000). Validación factorial del "General Health Questionnaire" (GHQ12) mediante análisis factorial confirmatorio. Psicología de la Salud, 12, 75-89.

Clemente, M., Reig-Botella, A., \& Coloma, R. (2015). The occupational health of correctional officers in Peru: The impact of length of work experience. The Prison Journal, 95(2), 244-263.

Diamond, P. M., Wang, E. W., Holzer, C. E. III, Thomas, C., \& Anges, C. des. (2001). The prevalence of mental illness in prison. Administration and Policy in Mental Health, 29, 21-40.

Diefendorff, J. M., Erickson, R. J., Grandey, A. A., \& Dahling, J. J. (2011). Emotional display rules as work unit norms: A multilevel analysis of emotional labor among nurses. 
Journal of Occupational Health Psychology, 16, 170-186.

Douglas, M. (1966). Purity and Danger: An Analysis of Concepts of Pollution and Taboo. London: Routledge.

Fuertes, F. C., Moreno, E. S., \& Urquiola, Y. A. (2017). Burnout en funcionarios/as de prisiones en Chile. Revista Iberoamericana de Psicología: Ciencia y Tecnología, 9(1), 63-72.

Gailliot, M. T., Baumeister, R. F., DeWall, C. N., Maner, J. K., Ashby Plant, E., Tice, D. M., ... Schmeichel, B. J. (2007). Selfcontrol relies on glucose as a limited energy source: Willpower is more than a metaphor. Journal of Personality and Social Psychology, 92, 325-336.

Goldberg, D. (1992). General Health Questionnaire (GHQ-12). Windsor, UK: NFER-Nelson.

Grandey, A., Chi, N. W., \& Diamond, J. A. (2013). Show me the money! Do financial rewards for performance enhance or undermine the satisfaction from emotional labor? Personnel Psychology, 66, 569-612.

Härenstam, A., Palm, U. B., \& Theorell, T. (1988). Stress, health and the working environment of Swedish prison staff. Work Eु Stress, 2(4), 281-290.

Harvey, J. (2014). Perceived physical health, psychological distress, and social support among prison officers. The Prison Journal, 94(2), 242-259.

Hobfoll, S. E. (1989). Conservation of resources: A new attempt at conceptualizing stress. American Psychologist, 44, 513-524.

Hülsheger, U. R., \& Schewe, A. F. (2011). On the costs and benefits of emotional labor: A meta-analysis of three decades of research. Journal of Occupational Health Psychology, 16, 361-389.

Jin, X., Sun, I. Y., Jiang, S., Wang, Y., \& Wen, S. (2016). The Impact of Job Characteristics on Burnout Among Chinese Correctional Workers. International Journal of Offender Therapy and Comparative Criminology. http:// dx.doi.org/10.1177/0306624X16648419
Kalra, R., Kollisch, S. G., MacDonald, R., Dickey, N., Rosner, Z., \& Venters, H. (2016). Staff satisfaction, ethical concerns, and burnout in the New York City Jail Health System. Journal of Correctional Health Care, 22(4), 383-392.

Kinman, G., Clements, A. J., \& Hart, J. (2016a). Work-related wellbeing in UK prison officers: A benchmarking approach. International Journal of Workplace Health Management, 9(3), 290-307.

Kinman, G., Clements, A. J., \& Hart, J. (2016b). Working conditions, work-life conflict, and well-being in UK prison officers: The role of affective rumination and detachment. Criminal Justice and Behavior. http:// dx.doi.org/10.1177/0093854816664923

Lamb, H. R., \& Weinberger, L. E. (1998). Persons with severe mental illness in jails and prisons: A review. Psychiatric Services, 49, 483-92.

Lambert, E. G., Barton-Bellessa, S. M., \& Hogan, N. L. (2015). The consequences of emotional burnout among correctional staff. Sage Open, 5(2). http:// dx.doi.org/10.1177/2158244015590444

Lambert, E. G., Minor, K. I., Wells, J. B., \& Hogan, N. L. (2015). Leave your job at work: The possible antecedents of workfamily conflict among correctional staff. The Prison Journal, 95 (1), 114-134.

Little, L. M., Kluemper, D., Nelson, D. L., \& Gooty, J. (2012). Development and validation of the interpersonal emotion management scale. Journal of Occupational and Organizational Psychology, 85, 407-420.

Martínez-Íñigo, D., \& Crego, A. (2010). Doing "dirty work" with severe mental disorder people: The role of interpersonal affect regulation in the normalization process. Comunicación presentada en el 70 Encuentro Anual de la Academy of Management, Montreal, Canadá.

Martínez-Íñigo, D., Mercado, F., \& Totterdell, P. (2015). Using interpersonal affect regulation in simulates health care consultations: An experimental 
investigation of self-control-resource depletion. Frontiers in Psychology, 6, 1-14.

Martínez-Íñigo, D., Poerio, G. L., \& Totterdell, P. (2013). The association between Controlled Interpersonal Affect Regulation and resource depletion. Applied Psychology: Health and Well-Being, 5, 248-269.

Martínez-Íñigo, D., \& Totterdell, P. (2016). The mediating role of distributive justice perceptions in the relationship between emotion regulation and emotional exhaustion in health care workers. Work and Stress, 1, 26-45

Martínez-Íñigo, D., Totterdell, P., Alcover, C. M., \& Holman, D. (2007). Emotional labour and emotional exhaustion: Interpersonal and intrapersonal mechanisms. Work and Stress, 21, 30-47.

Martínez-Íñigo, D., Totterdell, P., Alcover, C. M., $\&$ Holman, D. (2009). The source of display rules and their effects on primary health care professional's well-being. The Spanish Journal of Psychology, 12, 618-631.

Math, S. B., Murthy, P., Parthasarthy, R. C., Kumar, N., \& Madhusudhan, S. (2011). Minds Imprisoned: Mental Health Care in Prisons. Bangalore: National Institute of Mental Health Neuro Sciences.

Muraven, M., \& Baumeister, R. F. (2000). Self-regulation and depletion of limited resources: Does self-control resemble a muscle? Psychological Bulletin, 126, 247-259.

Naciones Unidas. (2009). Report of the Special Rapporteur on torture and other cruel, inhuman or degrading treatment or punishment, Manfred Nowak. Mission to Uruguay. Asamblea General de las Naciones Unidas, Consejo de Derechos Humanos, sesión 13. Recuperado de http://www2.ohchr.org/english/bodies/h rcouncil/docs/13session/A-HRC-13-39-Ad d2.pdf

Niven, K., Holman, D., \& Totterdell, P. (2012). How to win friendship and trust by influencing people's feeling: An investigation of interpersonal affect regulations and the quality of relationships. Human Relations, 65, 777-805.
Niven, K., Totterdell, P., \& Holman, D. (2007). Changing moods and influencing people: The use and effects of emotional influence behaviours at HMP Grendon. Prison Service Journal, 173, 39-45.

Niven, K., Totterdell, P., \& Holman, D. (2009). A classification of controlled interpersonal affect regulation strategies. Emotion, 9(4), 498-509.

Niven, K., Totterdell, P., Holman, D., \& Headley, T., (2012). Does regulating others' feelings influence people's own affective well-being? The Journal of Social Psychology, 152, 246-260.

Niven, K., Totterdell, P., Stride, C. B., \& Holman, D. (2011). Emotion regulation of others and self (EROS): The development and validation of a new individual difference measure. Current Psychology, 30, 53-73.

Roberts, S. E., Jaremin, B., \& Lloyd, K. (2013). High-risk occupations for suicide. Psychological Medicine, 43(06), 1231-1240.

Rodríguez Amaya, R. M. (2014). Burnout syndrome in prison guards, Bucaramanga, Colombia, 2013. Revista Colombiana de Psiquiatria, 43(3), 146-153.

Sandín, B, Chorot, P. Lostao, L., Joiner, T. E., Santed, M. A., \& Valiente, R. M. (1999). Escalas panas de afecto positivo y negativo: validación factorial y convergencia transcultural. Psicothema, 11, 37-51.

Schaufeli, W. B., van Dierendonck, D., \& van Gorp, K. (1996). Burnout and reciprocity: Towards a dual-level social exchange model. Work Eु Stress, 10, 225-237.

Steiner, B., \& Wooldredge, J. (2015). Individual and environmental sources of work stress among prison officers. Criminal Justice and Behavior, 42(8), 800-818

Stöver, H. (2017). Prison staff under stress: Causes, consequences and health promotion strategies. En B. Elger, C. Ritter, $\&$ H. Stöver (Eds.). Emerging Issues in Prison Health (pp. 253-259). Dordrecht: Springer.

Sui, G. Y., Hu, S., Sun, W., Wang, Y., Liu, L., Yang, X. S., \& Wang, L. (2014). Prevalence and associated factors of depressive symptoms 
among Chinese male correctional officers. International archives of occupational and environmental health, 87(4), 387-395.

Totterdell, P., Hershchovis, M. S., Niven, K., Reich, T. C., \& Stride C. (2012). Can employees be emotionally drained by witnessing unpleasant interactions between coworkers? A diary study of induced emotion regulation. Work E Stress, 26, 112-129.

Tyler, J. M., \& Burns, K. C. (2008). After depletion: The replenishment of the self's regulatory resources. Self and Identity, 7 , 305-321.

Viotti, S. (2016). Work-related stress among correctional officers: A qualitative study. Work, 53(4), 871-884.

Vohs, K. D., \& Baumeister, R. F. (2007). Selfregulation, ego-depletion and motivation. Social and Personality Psychology Compass, 10, 115-128.

Zaki, J., \& Williams, C. (2013). Interpersonal emotion regulation. Emotion, 13, 803-810.

\section{Notas}

* Artículo de investigación. 